\title{
Percepções e mudanças na qualidade de vida de pacientes submetidos à hemodiálise
}

\author{
Perceptions and changes in the quality of life of patients submitted to hemodyalisis
}

Percepciones y cambios en la calidad de vida de los enfermos sometidos a hemodiálisis

\author{
Alessandra Silva da Silva', Rosemary Silva da Silveira", Geani Farias Machado Fernandes', \\ Valéria Lerch Lunardi", Vânia Marli Schubert Backes" \\ ' Universidade Federal do Rio Grande, Escola de Enfermagem. Rio Grande-RS, Brasil. \\ "Universidade Federal do Rio Grande, Escola de Enfermagem, \\ Programa de Pós Graduação em Enfermagem. Rio Grande-RS, Brasil.
}

Submissão: 03-12-2009 Aprovação: 18-11-2011

\section{RESUMO}

A Insuficiência Renal Crônica é um importante problema de Saúde Pública. O tratamento dialítico, na espera pelo transplante renal, constitui-se numa expectativa de melhoria da qualidade de vida. Este estudo qualitativo, realizado em Rio Grande-RS, objetivou conhecer as percepções dos pacientes com Insuficiência Renal Crônica acerca das mudanças ocorridas em sua rotina de vida, decorrentes do tratamento de hemodiálise, identificando os elementos que influenciam a sua qualidade de vida. Os resultados evidenciaram que os sentimentos iniciais de indignação e negação se modificam, enquanto os pacientes se fortalecem para o enfrentamento da doença. Restrições dos hábitos alimentares e hídricos, incapacidade ou limitação das atividades físicas, profissionais e de lazer foram as principais dificuldades identificadas. O apoio dos familiares e dos profissionais da saúde pode contribuir para a superação dessas limitações e adaptação ao novo estilo de vida.

Descritores: Qualidade de vida; Insuficiência Renal Crônica; Hemodiálise; Cuidados de enfermagem.

\section{ABSTRACT}

Chronicle Renal Failure is considered a major problem in Public Health. The dyalitic treatment while waiting for the renal transplant means a boost on quality of life. This qualitative study, carried out in Rio Grande-RS, aimed at knowing the perceptions of patients with Chronicle Renal Failure, identifying the elements that influence their quality of life. The results highlighted that the initial feelings of indignation and denial change as they strengthen themselves to face the disease. Restrictions of fluid and eating habits, inability to perform or limitation in activities, professional and leisure, were the main difficulties identified. The support from family members and from health professionals may help to overcome these limitations and adaptation to a new lifestyle.

Key words: Quality of life; Chronicle renal failure; Hemodyalisis; Nursing care.

\section{RESUMEN}

La Insuficiencia Renal Crónica es un importante problema para la Salud Pública. El tratamiento dialítico, en la espera por el trasplante renal, se constituye en una expectativa de mejoría de la calidad de vida. Este estudio cualitativo, realizado en Rio Grande-RS, tuvo por objetivo conocer las percepciones de los enfermos con Insuficiencia Renal Crónica sobre los cambios ocurridos en su rutina de vida que derivan del tratamiento de hemodiálisis, identificando los elementos que influencian su calidad de vida. Los resultados evidenciaron que los primeros sentimientos de indignación y negación se modifican a la medida que se fortalecen para enfrentar a la enfermedad. Restricciones de los hábitos alimentares e hídricos, incapacidad o limitación de las actividades físicas, profesionales y de ocio fueran las principales dificultades identificadas. El apoyo de los parientes y de los profesionales de la salud puede contribuir para la superación de esas limitaciones y adaptaciones al nuevo estilo de vida. Palabras clave: Calidad de vida; Insuficiencia Renal Crónica; Hemodiálisis; Atención de enfermería. 


\section{INTRODUÇÃO}

A insuficiência renal crônica é considerada uma doença de elevada morbidade e mortalidade. Sua incidência e prevalência em estágio avançado têm aumentado no Brasil e, em todo mundo, a doença vem se tornando uma epidemia. O Sistema Único de Saúde (SUS) é responsável por 87,2\% do custo total da terapia de substituição renal (TSR). De acordo com a Sociedade Brasileira de Nefrologia (SBN), no Brasil, existem 684 centros de tratamento dialítico e, destes, 150 (21,9\%) estão localizados na região Sul. A taxa de mortalidade anual desses pacientes é de 15,2\%. As doenças que comumente levam à insuficiência renal crônica são a hipertensão arterial e o diabetes ${ }^{(1)}$.

Assim, o diagnóstico e o tratamento precoce de doenças crônicas que apresentem potencial para desencadear insuficiência renal, bem como a identificação de lesões em órgãos alvo e/ou complicações crônicas, constituem-se num verdadeiro desafio para o Sistema Único de Saúde (SUS), para os trabalhadores da saúde e para a sociedade. O investimento na prevenção dessas doenças e de suas complicações é decisivo, não só para reduzir os gastos com a saúde, em razão do alto grau de sofisticação em que se encontra a tecnologia assistencial, como também para proporcionar uma maior qualidade de vida da população ${ }^{(1,2)}$.

Dentre as diversas definições de qualidade de vida, pode-se citar a proposta pela Organização Mundial da Saúde, que compreende este conceito como a "percepção do indivíduo de sua posição na vida, no contexto de sua cultura e no sistema de valores em que vive e em relação a suas expectativas, seus padrões e suas preocupações" ${ }^{\prime \prime}$.

As mudanças no estilo de vida acarretadas pela insuficiência renal crônica e pelo tratamento dialítico ocasionam limitações físicas, sexuais, psicológicas, familiares e sociais, que podem afetar a qualidade de vida. Na vivência cotidiana com estes pacientes, os mesmos expressam sentimentos negativos, como medo do prognóstico, da incapacidade, da dependência econômica e da alteração da autoimagem. Por outro lado, eles também reconhecem que o tratamento Ihes possibilita a espera pelo transplante renal e, com isso, uma expectativa de melhorar sua qualidade de vida. As mudanças decorrentes do tratamento atingem seus familiares, pois esses necessitam ajustar sua rotina diária às necessidades de apoio ao familiar que apresenta insuficiência renal crônica. Desse modo, faz-se necessário que os trabalhadores da saúde e da Enfermagem, em particular, considerem a relevância dessas questões na sua abordagem e na elaboração do seu plano de cuidados.

Neste sentido, a Enfermagem vem desenvolvendo pesquisas $^{(4,5)}$ voltadas para a melhoria da qualidade de vida de pacientes acometidos por doenças crônicas, acompanhando a tendência da área da saúde, pois, além do esforço e investimento direcionados ao aumento de anos de vida, com êxito, faz-se necessária a preocupação com a qualidade na vida aos anos a mais que foram conquistados.

Para tanto, acredita-se ser necessário atuar de modo mais próximo a estes pacientes; conhecer suas percepções frente às limitações enfrentadas e ao tratamento dialítico; descobrir os possíveis comprometimentos decorrentes destas situações, bem como as adaptações necessárias em suas vidas para a concretização do tratamento.

Assim, teve-se como objetivo deste estudo conhecer as percepções dos pacientes com insuficiência renal crônica acerca das mudanças ocorridas em sua rotina de vida decorrentes do tratamento de hemodiálise, identificando os elementos que influenciam a sua qualidade de vida.

A continuidade da realização de pesquisas acerca desta temática faz-se necessária, pois, apesar da hemodiálise ser utilizada no país desde a década de 1950, constata-se um desconhecimento da população em geral tanto sobre a prevenção da insuficiência renal crônica quanto sobre a hemodiálise. Dar voz aos sujeitos que vivenciam a doença, compreendendo seus anseios e necessidades é de extrema relevância para que a Enfermagem e demais profissionais de saúde possam cuidar deles e auxiliá-los a viver com a máxima qualidade possível, apesar das limitações da doença e do próprio tratamento.

\section{METODOLOGIA}

A busca do conhecimento das percepções dos pacientes renais crônicos submetidos à hemodiálise exige uma aproximação maior com os sujeitos do estudo, possibilitando o compartilhar de significados, crenças, valores, modos de viver e de enfrentar o tratamento dialítico. Por isso nossa opção por um estudo de natureza qualitativa, o qual foi realizado num Centro de Nefrologia e Diálise (CND) de um hospital localizado na cidade do Rio Grande-RS.

O CND atende uma demanda de aproximadamente 45 pacientes em programa de hemodiálise pelo SUS. Dentre esses pacientes, 15 apresentavam Hepatite $\mathrm{C}$ e três, o vírus da imunodeficiência humana HIV. A instituição possui ainda um ambulatório de nefrologia, no qual atuam médico e enfermeiro especializados, com uma média de 70 a 80 consultas/mês.

Os sujeitos deste estudo foram nove pessoas com IRC, que realizavam hemodiálise neste serviço. Destes, cinco (5) eram do sexo feminino e quatro (4) do sexo masculino, com idades entre 40 e 88 anos. O número de participantes foi definido mediante o interesse e adesão ao estudo, e quando a coleta de dados deixou de produzir novas informações, tornando-se redundante ${ }^{(6)}$.

A técnica de coleta de dados utilizada foi a entrevista, que ocorreu antes do início da sessão de hemodiálise, em uma sala apropriada, visando manter a privacidade dos sujeitos. Foram coletados dados relacionados às suas percepções e vivências com a doença e o tratamento de hemodiálise e as possíveis influências decorrentes deste processo. As entrevistas foram registradas por gravações em fita cassete, transcritas e analisadas segundo o método proposto por Minayo ${ }^{(7)}$.

De acordo com o estabelecido na Resolução $N^{\circ}$ 196/96 $6^{(8)}$, sobre Pesquisa Envolvendo Seres Humanos, este estudo ocorreu após aprovação do Comitê de Ética em Pesquisa na Área da Saúde da FURG/CEPAS, conforme o Processo $\mathrm{n}^{\circ}$ 23116.000814/2008-16 e Parecer $n^{\circ}$ 10/2008. Para manter o anonimato dos sujeitos entrevistados, os mesmos foram identificados pelos códigos P1 a P9. 


\section{RESULTADOS E DISCUSSÃO}

As categorias emergidas a partir da análise dos dados foram as percepções sobre a doença e as mudanças ocorridas no processo de viver dos pacientes com insuficiência renal crônica em decorrência do tratamento dialítico. Buscou-se, ainda, realizar uma síntese da influência destas percepções e das mudanças na qualidade de vida.

\section{As percepções do impacto da doença e do tratamento dialítico}

A insuficiência renal crônica representa a perda gradativa, lenta e progressiva da função de alguns néfrons, mantendo outros, com suas funções em condições adequadas até a irreversibilidade do comprometimento das funções renais. É também uma condição silenciosa, pois o paciente só começa a perceber que apresenta alguma alteração renal quando inicia o aparecimento dos sintomas urêmicos, ou seja, quando os rins perdem aproximadamente $50 \%$ de sua função ${ }^{(1)}$.

A partir dos relatos dos sujeitos da pesquisa e das experiências vivenciadas no CND, foi possível perceber que, na maioria dos casos, o paciente só descobre a doença quando aparecem os sintomas de uremia e da Insuficiência Renal Crônica (IRC), ou seja, quando se encontra num estágio tardio, necessitando de um processo dialítico.

Eu sentia muita coceira no corpo, sentia a minha cabeça sempre pesada, mas eu nunca medi a pressão [...] eu vim fazer o exame [...] passou uns dois, três dias e me chamaram aqui e disseram que eu já tava com problema nos rim, um já estava parado e o outro estava parando e eu tinha urgentemente que entrar nas máquinas, eu internei e no outro dia já fiz fístula, já coloquei o cateter e já entrei direto e a partir dali não parei mais, já fui direto pra máquina, sentia falta de ar, agora não sinto mais [...] (P1).

A realização do diagnóstico precoce de Insuficiência Renal é fundamental, pois, quando ocorre tardiamente, a função renal já está comprometida, desencadeada por um quadro avançado, com a necessidade de um tratamento dialítico, conforme expresso nas falas dos sujeitos. Na maioria das vezes, os primeiros sintomas perceptíveis são: edema dos olhos, pés e pernas; diurese espumosa; presença de anemia intensa, cansaço, sensação de fraqueza e aumento da pressão arterial.

Esta descoberta tardia deve-se à fragilidade de conhecimento da população quanto à prevenção e, pode-se dizer, à fragilidade da atenção em saúde oferecida pelos profissionais de saúde, em relação aos esclarecimentos acerca da relevância da preservação da função renal para o nosso corpo, dos cuidados necessários com o sistema urinário, dos riscos presentes em afecções urinárias. Considerando-se o trabalho da Enfermagem e, especificamente, da enfermeira nas unidades de saúde do SUS no processo de educação em saúde, suas possibilidades de atuação são extremamente importantes por sua proximidade com os usuários, com o seu modo de viver e de se cuidar, não apenas podendo, mas devendo fazer diferenças nos espaços profissionais ocupados, em especial, quanto à prevenção de doenças e, entre essas, a doença renal.

Ações de educação em saúde direcionadas aos portadores de insuficiência renal (IR), para evitar que o diagnóstico da doença ocorra já em um estágio avançado em que há a necessidade de um tratamento dialítico e seu impacto na vida dos pacientes, poderiam favorecer a conscientização da população da necessidade de prevenção, uma vez que essa doença, além de trazer consequências físicas ao indivíduo que a vivencia, traz prejuízos psicológicos e altera o seu cotidiano, sendo caracterizada também como um problema social, por interferir no papel que esse indivíduo desempenha na sociedade $^{(10)}$.

Após a descoberta do diagnóstico de IRC, os pacientes passam por um processo de rejeição/aceitação frente à necessidade do tratamento dialítico, podendo apresentar diferentes reações e modos de agir durante o processo de enfrentamento.

Muito complicado a aceitação, primeiro tu tem que trabaIhar a tua cabeça que a tua vida mudou drasticamente [...] nem sabia que existia a diálise, pra mim foi um susto, o que é isso? Eu não sabia da existência disso, [...] eu tenho certa consciência que é bastante complicado fazer hemodiálise, mas é um mal necessário hoje para várias pessoas (P2).

A hemodiálise acarreta sentimentos ambíguos de aceitação e revolta nos sujeitos que necessitam deste tratamento para sobreviver, pois ao mesmo tempo em que garante a vida, torna a pessoa dependente da tecnologia. Há um simbolismo atribuído à hemodiálise, tratando a mesma como uma relação de "vida e morte", considerando que a sobrevivência é possível pelo procedimento de hemodiálise ${ }^{(10)}$.

Os sentimentos relatados pelos pacientes incluem angústia, insegurança, pânico, depressão, desânimo, sensação de prisão da máquina, medo relacionado às limitações decorrentes desta situação e das suas repercussões e modificações no modo de ser e viver, com possíveis alterações em sua qualidade de vida. Esta idéia é confirmada por Sisdelli ${ }^{(11)}$, quando refere que

O primeiro sintoma de quem se encontra "doente" é o desânimo. Não existe vontade de falar pensar e muito menos agir. É como se nosso cérebro murchasse. Acredito que todo "doente renal crônico" passa por essa fase de apatia [...]. Onde se encontra o lado positivo, ficando preso a uma máquina, três vezes por semana durante quatro horas [...], não era nem drama, mas uma verdadeira tragédia, porque me encontrava mais preparada para "o outro lado da vida" do que para o tratamento, tal era o desânimo que me perseguia. [...] ao iniciar o tratamento, a melhora começou a ser rápida [...] surpreendente. O desânimo foi o primeiro a desaparecer e o cérebro voltou ao normal.

A descoberta de uma doença crônica e a necessidade do tratamento dialítico podem, inicialmente, ser encaradas como uma dificuldade, ocasionando sofrimento físico e psíquico aos pacientes. A mudança brusca no seu viver, o convívio com as limitações, o enfrentamento da hemodiálise como uma necessidade contínua e a possibilidade da morte, podem 
influenciar negativamente a sua qualidade de vida. Destaca-se a relevância do profissional enfermeiro instrumentalizar-se, não apenas no domínio das tecnologias que envolvem o procedimento em si, mas, considerar e incluir no planejamento do cuidado de enfermagem, os aspectos relacionados à dimensão emocional e social para contemplar as reais necessidades desses pacientes.

Percebeu-se que estes sentimentos se modificam durante o processo de conhecimento acerca da hemodiálise, podendo desaparecer ao longo do tratamento, tornando as sessões menos dolorosas e compreendidas como fundamentais para a vida dos pacientes com IRC.

Eu não aceitava, eu me desanimava, ficava pra baixo. [...] ter que vir, não poder faltar, [...] agora não, agora eu to bem, aceitei bem, se não for assim o prejuízo é só meu (P3).

Não vou te dizer que não dói as agulhas, dói as agulhas, tem horas que dói, dói pra ligar, dói pra desligar, mas é uma oportunidade pra ti viveres mais um pouco (P4).

O contexto que envolve o tratamento hemodialítico, por si só, já denota mecanicidade. Preocupar-se com a segurança do paciente ao longo da realização do tratamento evitando complicações é uma constante no cotidiano dos trabalhadores da Enfermagem $^{(12)}$. No entanto, o profissional de enfermagem, ao prestar cuidado ao paciente submetido a este tratamento, deve atentar para que suas ações não se transformem num fazer automático, sendo necessário valorizar os aspectos humanos na relação cuidador/cuidado.

\section{As mudanças ocorridas no processo de viver dos pacien-} tes submetidos ao tratamento dialítico

A IRC e a necessidade de realizar um tratamento dialítico provocam sucessivas mudanças na vida dos pacientes, tanto físicas, quanto psicológicas e sociais. Há a necessidade de mudar hábitos na alimentação: evitar o sal, a gordura e o excesso de líquidos, exigindo um controle que antes não era realizado por estes pacientes, conforme evidenciado abaixo:

Não posso tomar muito líquido, o sal também eu não posso comer [...] tenho algumas limitações que eu não tinha antes agora eu tenho (P1)

A modificação de hábitos alimentares e hídricos foi necessária para que estes pacientes melhorassem sua qualidade de vida. Para tanto, foi fundamental a ação educativa da equipe de saúde esclarecendo os questionamentos relativos à dieta e suas restrições; o controle da ingestão hídrica e, também, os nutrientes indispensáveis para uma alimentação equilibrada e adequada ao quadro clínico ${ }^{(13)}$.

Essas mudanças nos hábitos de vida não se restringem à alimentação e hidratação. Quando se apresenta uma doença crônica, a necessidade de mudanças pode se estender a hábitos relacionados com a atividade física, lazer e trabalho. Além disso, o paciente permanece dependente de tecnologias, podendo ser necessária a utilização contínua de medicações, assim como a dependência de familiares, de profissionais de saúde e ou de outros cuidadores. Assim, a vida pode tornar-se um desafio ${ }^{(9,13)}$.

A restrição física de um dos braços, em decorrência da fístula arteriovenosa (FAV), e do desconforto causado pelo cateter no pescoço provocou mudanças no desempenho das atividades diárias e profissionais dos pacientes, tornando-os mais inseguros no cuidado consigo mesmo. Alguns sujeitos têm, também, sua autoimagem alterada, como se pode perceber nos depoimentos a seguir.

O que me atrapalha é essa fístula aqui que parou e ai tem que fazer de novo, usar essa coisa aqui no pescoço, não me sinto bem com isso aqui (P1)

A fístula eu procuro cuidar sempre dentro do possível, não vou carregar muito peso com o braço [...] eu tenho medo de machucar, esteticamente não é uma coisa legal, tu aprende e relevar muitas coisas (P2)

As limitações decorrentes da imagem que fazem do seu corpo pela presença da fístula e a dependência da tecnologia precisam ser desmistificadas pela equipe de saúde. Os pacientes reconhecem a necessidade da FAV para a efetivação do tratamento, porém relatam sentimentos como tristeza, amargura e dependência ${ }^{(14)}$; além dos cuidados com a qualidade de acesso e manutenção da FAV, é necessário propiciar um espaço terapêutico para que expressem seus sentimentos e dúvidas. É fundamental discutir com estes pacientes os cuidados com a mesma, a fim de evitar limitações desnecessárias. A construção de grupos para a troca de experiências entre si pode favorecer a superação das limitações.

Uma das mudanças ocorridas no processo de viver dos pacientes em tratamento dialítico, ressaltadas por eles e que afetaram sobremaneira a sua qualidade de vida, refere-se às atividades profissionais.

Uma pessoa como eu, que tinha uma atividade física muito intensa; eu era Profa. de Ed. Física. Então, foi uma coisa bastante complicada, eu ia pra academia, fazia aula de dança, era a minha vida, era a minha grande realização[...] hoje eu me acho meio inútil, às vezes eu to em casa, eu tenho tempo demais, tem tempo livre demais e, ao mesmo tempo, não consigo achar nada que me ocupe e que eu me sinta legal [...] pra mim foi muito complicado, de uma hora para outra tu te vê fraca por causa da anemia, sem poder fazer várias coisas que tu fazia, presa numa máquina pra poder sobreviver (P3).

A interrupção das atividades profissionais em decorrência do diagnóstico e do tratamento dialítico, na visão destes pacientes, foi influenciada por vários fatores, dentre os quais ressaltam o fato de ter que se submeter às sessões de tratamento dialítico, de modo contínuo, por três dias na semana, necessitando ausentar-se do seu local de trabalho repetidamente e por muito tempo.

Além do trabalho, essas mudanças atingem outras dimensões da vida social, pois as relações sociais que estabeleciam 
no ambiente de trabalho, nas atividades de lazer e nas viagens são alteradas pelo tratamento. A alegria de viver associada ao prazer de viajar, de visitar a família, valorizada por eles como importantes para manter sua qualidade de vida, ficam comprometidas, inicialmente, por sentirem-se inseguros com o cuidado com a fístula e, também, por estarem receosos em realizar o tratamento dialítico em outra instituição de saúde com uma equipe desconhecida da sua, em outra cidade. Posteriormente, quando se habituam ao tratamento, passam a superar as limitações e os desconfortos ocasionados pelo tratamento, realizando as adaptações necessárias, e readequando suas atividades de acordo com que lhes é possível como portadores de uma doença crônica.

O trabalho foi destacado como importante em suas vidas, tanto pela satisfação de suas necessidades de sobrevivência, quanto pela realização por desempenharem atividades que Ihes proporcionavam prazer e satisfação. Pudemos compreender também que o fato de não trabalharem pode afetar outras dimensões da sua vida, ocasionando sentimentos de incapacidade e de limitações conseqüentes às alterações decorrentes da doença.

O trabalho, portanto, é determinante no equilíbrio psicológico do ser humano, à medida que o mantém solidamente vinculado à realidade, constituindo muitas vezes, fator norteador da vida humana, com implicações diretas nas condições fisiológicas, psíquicas, mentais e sociais do indivíduo ${ }^{(15:)}$. Assim é preciso uma atenção especial dos profissionais de saúde, não apenas em relação ao aspecto financeiro decorrente da ausência do trabalho, mas também aos outros problemas envolvidos como: o sentimento de inutilidade, ociosidade, desvalorização, e ainda a sensação de ser "um peso" para seus familiares.

As mudanças ocorridas no processo de viver dos pacientes identificadas neste estudo são também evidenciadas nas alterações relativas ao funcionamento social e papel ocupacional afetando em maior ou menor grau a qualidade de vida dos pacientes em tratamento dialítico ${ }^{(2)}$.

\section{A influência e o impacto do tratamento dialítico na qua- lidade de vida}

O tratamento dialítico, para estes sujeitos, configura-se tanto como um fator limitador da sua qualidade de vida, por ocasionar modificações e limitações em suas rotinas diárias, quanto como um fator potencializador, na medida em que avaliam o impacto do tratamento na melhoria das suas condições de vida e comparam o seu estado de saúde atual, com os problemas de saúde anteriormente apresentados.

Eu acho que a minha qualidade de vida melhorou [...] eu posso fazer meus serviços normais, não preciso ser carregada por ninguém, antes eu não podia subir nem num carro, quer dizer que a minha qualidade de vida melhorou, isso com certeza melhorou muito (P7)

A qualidade de vida para estes sujeitos relaciona-se ao apoio da família e à sensação de bem-estar posterior à realização das sessões de hemodiálise. O suporte familiar é decisivo na percepção dos sujeitos frente à doença crônica e na manutenção do tratamento. Neste sentido, apesar do diagnóstico de uma doença crônica e do tratamento dialítico fragilizar e provocar angústia em familiares e pacientes, a família ocupa um papel de destaque contribuindo para que o paciente se sinta protegido, menos inseguro, amado e significativo, sentimentos que, na maioria das vezes, atuam como estímulos positivos para o enfrentamento da doença ${ }^{(16)}$ e do seu tratamento. Assim, a importância do apoio familiar parece decisiva para uma avaliação positiva dos pacientes sobre sua qualidade de vida, o que deve ser considerado pelos profissionais de saúde e de enfermagem para amenizar o impacto do tratamento dialítico na qualidade de vida dos pacientes e da própria família.

Ainda, além do apoio familiar, a sensação de bem-estar oriunda do tratamento auxilia no enfrentamento das restrições e dos sentimentos negativos, necessitando uma tomada de consciência de sua condição de saúde para a valorização do tratamento como possibilidade de sentir-se bem e melhorar sua qualidade de vida. Apesar dos avanços da medicina, do aumento da expectativa de vida, o enfrentamento e a possibilidade de melhorar a qualidade de vida dependem da mudança dos conceitos e pré-conceitos dos pacientes e acompanhantes com relação a essa doença e à hemodiálise $\mathrm{e}^{(11)}$.

No planejamento de um cuidado mais efetivo, a enfermagem necessita valorizar a individualidade e a humanização do atendimento dos pacientes que se submetem à hemodiálise, incluindo ações que estimulem sua participação ativa em atividades de promoção à saúde, além de atuar junto à sociedade desmistificando e dirimindo dúvidas acerca desse tratamento $^{(13)}$.

O fato de ser um doente renal, ou de necessitar de tratamento dialítico, pode ser considerado inicialmente como provocador de sofrimento físico e psíquico, especialmente, no momento da descoberta do diagnóstico. A maioria dos pacientes avalia a realização do tratamento dialítico como satisfatória e como a possibilidade de obter uma maior qualidade de vida, apesar da dificuldade de alguns em reconhecer esse tratamento como uma necessidade. Para cada pessoa, há uma forma de operacionalizar sua avaliação, e a avaliação de um mesmo indivíduo pode variar com o tempo, com o estabelecimento de prioridades ao longo da vida e com as circunstâncias pelas quais a vida pode se modificar ${ }^{(17)}$.

Para tanto, é necessário ampliar o leque de atenção e extrapolar a fronteira da sofisticação tecnológica e do aparato instrumental que, mesmo sendo importantes e indispensáveis no tratamento hemodialítico, são insuficientes para suprir as necessidades do ser humano que está sendo cuidado ${ }^{(18)}$.

\section{CONSIDERAÇÕES FINAIS}

O conhecimento técnico-científico, os avanços e descobertas em relação ao tratamento dialítico são fundamentais, mas, tão importante quanto as possibilidades de tratamento, é a sensibilidade do profissional de saúde, principalmente da Enfermagem, pois é a que permanece a maior parte do tempo com o paciente, em auxiliá-lo na redução da tensão e manutenção do equilíbrio emocional, facilitando a adaptação ao 
novo estilo de vida decorrente da doença e do tratamento. Para isso é preciso exercitar a escuta e considerar a percepção dos pacientes, suas necessidades e seus sentimentos em relação ao tratamento dialítico.

Apesar dos diferentes sentimentos de frustração, indignação e negação frente à necessidade do tratamento, especialmente no seu início, foi possível perceber que estes se modificam durante o processo de conhecimento e enfrentamento da doença pela hemodiálise. Dentre a sucessão de mudanças ocorridas na rotina de vida dos sujeitos, foram citadas: as restrições dos hábitos alimentares e hídricos, a incapacidade ou a limitação das atividades profissionais, físicas e de lazer. Entretanto, quando os pacientes recebem o apoio de seus familiares e dos trabalhadores da saúde conseguem melhor superar essas limitações e se readaptar à nova rotina de vida, reconhecendo o tratamento como decisivo para a sua qualidade de vida. Assim, entende-se que a aceitação do tratamento dialítico e as readaptações necessárias nas rotinas e estilo de vida são necessárias para um viver com mais qualidade.

Os trabalhadores da saúde precisam estar atentos às necessidades dos pacientes e familiares, para que possam, através do diálogo, apoiá-los na resolução das dificuldades encontradas percebendo a magnitude da presença de um ser humano que almeja ver atendido ou, pelo menos, ser compreendido em suas expectativas e ser respeitado em sua individualidade. Ações educativas para a promoção da saúde e para assegurar a qualidade de vida necessitam estar dirigidas para socializar o saber já disponível para favorecer a construção de novas maneiras de viver melhor, apesar das limitações impostas pela doença e o tratamento dialítico.

\section{REFERÊNCIAS}

1. Lehmkuhl A, Maia AJM, Machado MO. Estudo da prevalência de óbitos de pacientes com doença renal crônica associada à doença mineral óssea. J. Bras. Nefrol. 2009;31(1):10-17

2. Cruz LN, Polanczyk CA, Fleck MPA. Qualidade de vida em insuficiência renal crônica. In: Fleck MPA. A avaliação de qualidade de vida: guia para profissionais de saúde. Porto Alegre: Artmed; 2008. p. 208-17.

3. The Whoqol Group. The World Health Organization Quality of Life assessment (Whoqol): position paper from the World Health Organization. Social Sci Med. 1995;41(10):1403-9.

4. Martins MRI, Cesarino CB. Qualidade de vida de pessoas com doença renal crônica em tratamento hemodialítico. Rev. Latino-Am. Enfermagem 2005;13(5):670-6.

5. Martins LM, França APD, Kimura M. Qualidade de vida de pessoas com doença crônica. Rev. Latino-Am. Enfermagem 1996;4(3):5-8.

6. Polit DF, Hungler BP. Fundamentos de pesquisa em enfermagem. Porto Alegre: Artes Médicas; 1995.

7. Minayo MCS. O desafio do conhecimento: pesquisa qualitativa em saúde. 10ª ed. São Paulo: Hucitec; 2007.

8. Ministério da Saúde (Brasil). Conselho Nacional de Saúde. Resolução n. 196/96. Dispõe sobre pesquisa envolvendo seres humanos. [online] 1996 out 10 [citado em 12 jul 2009]. Disponível em: URL: http://www.ufrgs.br/ bioetica/res19696.htm

9. Dyniewicz AM, Zanella E, Kobus LSG. Narrativa de uma cliente com insuficiência renal crônica: a história oral com estratégia de pesquisa. Rev. Eletr. Enf. [periódico online] 2004 [citado em 20 abr 2008]; 6(2):199-212. Disponível em: http://fen.ufg.br

10. Reis CK, Guirardello EB, Campos CJG. O indivíduo renal crônico e as demandas de atenção. Rev. Bras. Enferm. [periódico online] 2008 jun [citado em 24 ago 2009]; 61(3):336-341. Disponível em: http://www.scielo.br/pdf/ reben/v61n3/a10v61n3.pdf

11. Sisdelli S. Diálise: vida nova ou nova vida? São Paulo: O Mercador Criação Contemporânea; 2007.

12. Nascimento CD, Marques IR. Intervenções de enfermagem nas complicações mais freqüentes durante a sessão de hemodiálise: revisão da literatura. Rev Bras Enferm [periódico online] 2005 dez [citado em 24 ago 2009]; 58(6):719-722. Disponível em: http://www.scielo.br/pdf/ reben/v58n6/a17v58n6.pdf

13. Campos CJG, Turato ER. Tratamento hemodialítico sob a ótica do doente renal: estudo clínico qualitativo. Rev Bras Enferm 2010;63(5):799-805.

14. Koepe GBO, Araújo STC. A percepção do cliente em hemodiálise frente à fistula artério venosa em seu corpo. Acta paul. enferm. 2008;21(esp):147-51.

15. Carreira L, Marcon SS. Cotidiano e trabalho: concepções de indivíduos portadores de insuficiência renal crônica e seus familiares. Rev. Latino-Am. Enfermagem 2003;11(6):823-31.

16. Silveira RS, Lunardi VL, Lunardi WD, Oliveira AMN. Uma tentativa de humanizar a relação da equipe de enfermagem com a família de pacientes internados na UTI. Texto Contexto Enferm 2005;14 (esp):125-30.

17. Fernandes GFM. Qualidade de Vida: representações sociais de docentes de enfermagem [tese]. Florianópolis: Curso de Pós-Graduação em Enfermagem, Universidade Federal de Santa Catarina; 2007.

18. Pietrovsk V, Dall'Agnol CM. Situações significativas no espaço-contexto da hemodiálise: o que dizem os usuários de um serviço? Rev Bras Enferm [online] 2006 out [citado 2009 ago 24]; 59(5):630-635. Disponível em: URL: http:// www.scielo.br/pdf/reben/v59n5/v59n5a07.pdf 